\title{
Hidden Moyamoya Disease Mimicking Reversible Cerebral Vasoconstriction Syndrome
}

Jae Jung Lee, Hong Kyun Park, Joong Yang Cho

Department of Neurology, Ilsan Paik Hospital, Inje University College of Medicine, Goyang, Korea

Moyamoya disease (MMD) is a progressive steno-occlusive disorder of the cerebral vasculature around the circle of Willis with secondary evolution of collateral vessels. In a few reported cases, MMD mimicked reversible cerebral vasoconstriction syndrome (RCVS). A 29-year-old woman experienced a sudden-onset generalized tonic-clonic seizure in the postpartum period. The initial brain magnetic resonance image showed high signal intensities in the bilateral basal ganglia and corona radiata on $\mathrm{T} 2$-weighted images along with severe stenosis in the bilateral distal internal carotid arteries and proximal middle and anterior cerebral arteries. The diagnosis of posterior leukoencephalopathy syndrome (PLS) combined with RCVS was established. Follow-up computed tomography angiography performed after 12 weeks showed comparable persistent stenosis without improvement. Thereafter, the diagnosis of MMD was highly suspected and further clinical history identified the same disorder in her father. Here we report a confusing case of PLS with hidden MMD mimicking RCVS.

J Neurosonol Neuroimag 201\$;10(2):162-165

Key Words: Moyamoya disease; Posterior leukoencephalopathy syndrome; Reversible cerebral vasoconstriction syndrome

Received: November 27, 2018 Revised: December 11, 2018 Accepted: December 11, 2018

Address for correspondence: Jae Jung Lee

Department of Neurology, Ilsan Paik Hospital, Inje University College of Medicine, 170 Juhwaro, Ilsanseo-gu, Goyang 10380, Korea

Tel: +82-31-910-7882

Fax: $+82-31-910-7319$

E-mail:leejto@naver.com
Making an accurate diagnosis of intracranial arterial stenosis in young adults is somewhat challenging because of its heterogenous collection of etiologies. They might include various disorders such as arterial dissection, Moyamoya disease (MMD), vasculitis, fibromuscular dysplasia, reversible cerebral vasoconstriction syndrome (RCVS), and common simple atherosclerosis. MMD refers to a disorder or syndrome with chronic progressive steno-occlusion of the vasculature around the circle of Willis followed by the evolution of prominent collateral vascular circulations. The diagnosis of MMD occasionally causes concern in cases of early-stage patient whose vessel stenoses are subtle and merely confined to the proximal parts of the major arteries. RCVS is a vascular disorder characterized by multifocal cerebral vasoconstrictions in conjunction with clinical thunderclap headache or seizures. It might present with characteristics similar to those of MMD, but it usually resolves within 12 weeks of symptom onset. Here, we report a case of MMD with posterior leukoencephalopathy syndrome (PLS) initially confused with RCVS.

\section{CASE REPORT}

A 29-year-old woman experienced a sudden-onset generalized tonic-clonic seizure 5 hours after a normal vaginal delivery. There were no remarkable events including headache during the delivery process other than occasional elevation of the systolic blood pressure up to $150 \mathrm{mmHg}$ and a blood loss of approximately $500 \mathrm{~mL}$. She had a past history of epilepsy that first occurred at 2 years of age, was quite well controlled with antiepileptic medications, and for which treatment was terminated at 16 years of age. She was fully awake and 
able to communicate at the initial visit to our emergency service. A comprehensive neurologic examination elicited no abnormal features. Laboratory tests including a thyroid function test were unremarkable other than mild creatine kinase elevation to $303 \mathrm{U} / \mathrm{L}$ (reference range, $<170)$. Brain magnetic resonance imaging and magnetic resonance angiography (MRA) showed high signal intensities in the bilateral basal ganglia and posterior parietal regions in T2 and fluid-attenuated inversion recovery images along with severe stenosis in the bilateral distal internal carotid arteries (ICA) and proximal middle arteries (MCA) and anterior cerebral arteries (ACA) (Fig. 1). Diffusion-weighted imaging revealed no features of diffusion restriction. According to those clinical and imaging findings, a diagnosis of postpartum PLS combined with RCVS was considered first. She was discharged on the day after conservative treatment with verapamil without any neurologic or systemic problems. Brain computed tomographic angiography (CTA) taken 12 weeks later, however, showed persistent prior stenosis with no improvements in the concordant regions (Fig. 2A, B) but normal densities of the bilateral basal ganglia and posterior parietal lobe. Thereafter, underlying hidden MMD was highly sus-
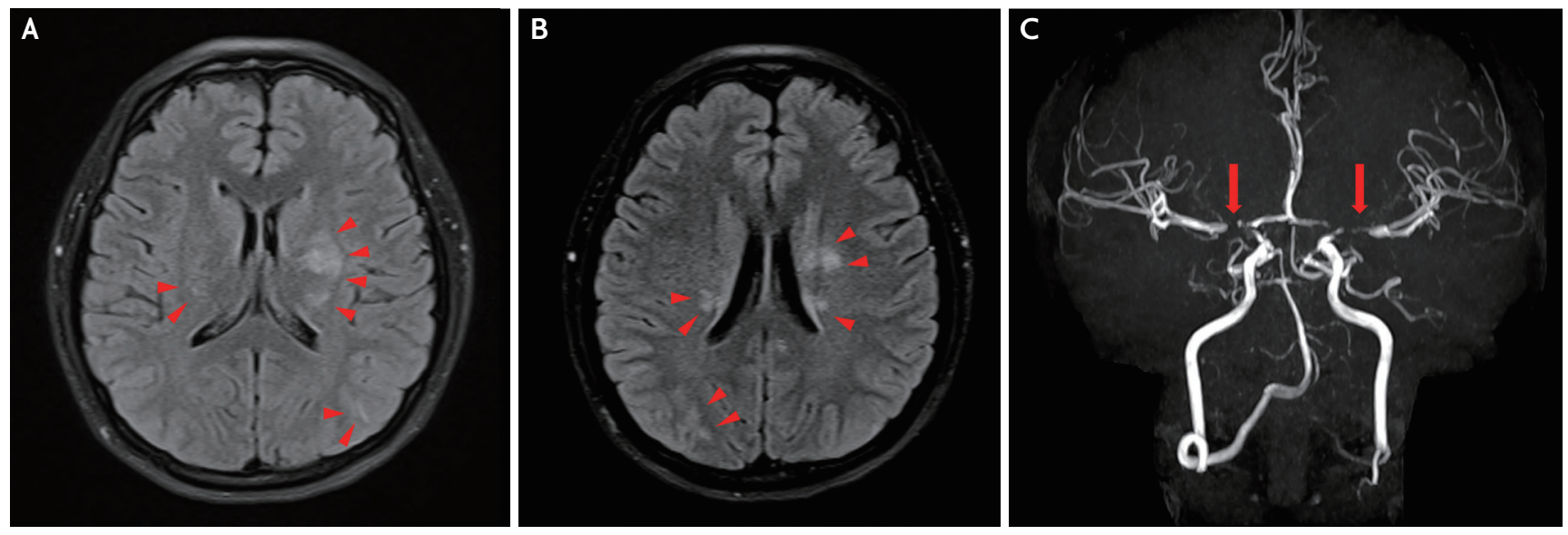

FIG. 1. Initial brain magnetic resonance imaging and angiography. (A, B) Mild high signal intensities in the region of bilateral basal ganglia and parietal lobes (arrowheads) in axial fluid-attenuated inversion recovery images. (C) Severe stenosis in the bilateral distal internal carotid arteries and the proximal middle and anterior cerebral arteries (arrows).
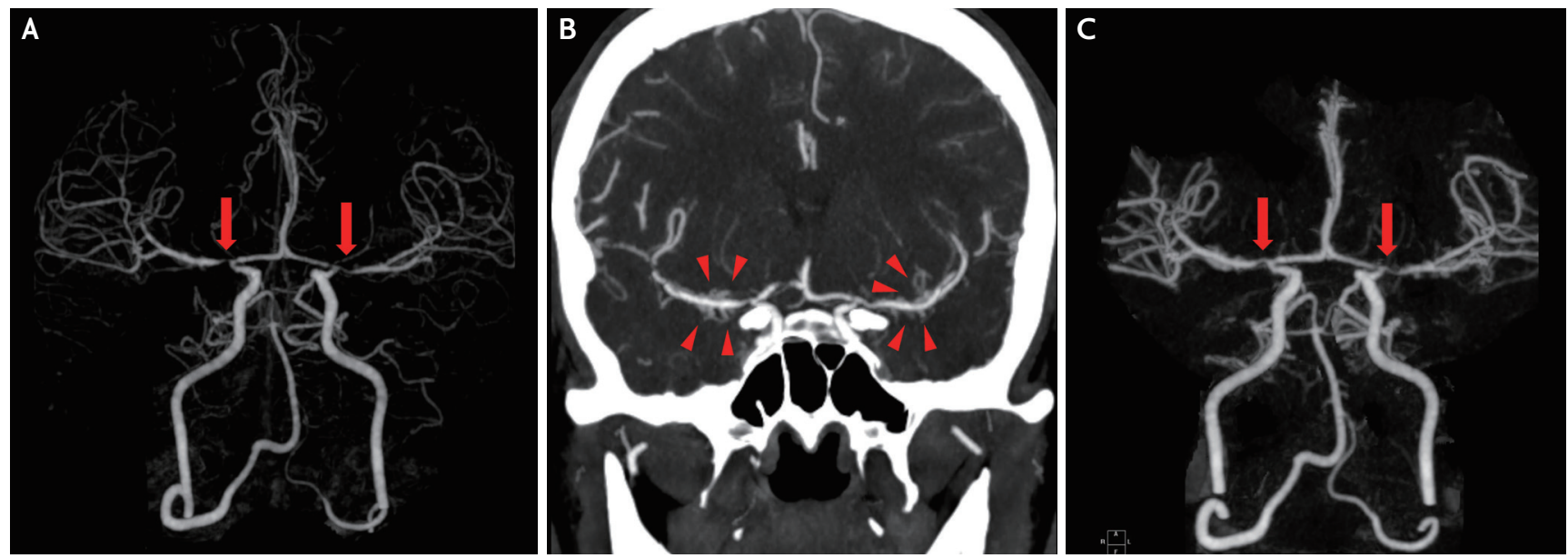

Fig. 2. Follow-up computed tomography angiography. (A) Persistent prior stenosis without any improvement in the bilateral distal internal carotid arteries and proximal middle and anterior cerebral arteries (arrows) as well as (B) suspicious collateral blood vessels (arrowheads) in the region of the bilateral middle cerebral arteries 12 weeks after baseline. (C) No interval changes in the stenoses in the concordant regions (arrows) an additional 6 months later. 
pected, and the further collection of the patient's family history revealed MMD in her father. Repeated CTA taken 6 months later presented an identical degree of stenosis in the same sate (Fig. 2 C); thus, the diagnosis of PLS with hidden MMD mimicking RCVS was ultimately made.

\section{DISCUSSION}

RCVS is often confused with another neurovascular disorder such as intracranial atherosclerosis, vasculitis, infectious arteritis, MMD, fibromuscular dysplasia, or another arterial disorder. Primary angitis of the central nervous system, which shows very similar clinical manifestations and overlapping radiologic findings with RCVS, has often been misdiagnosed over the past several decades. In addition, secondary inflammation within a constricted site may lead to irreversible and persistent vessel narrowing, ${ }^{1}$ which further complicates the precise diagnosis of RCVS. PLS is occasionally accompanied by RCVS, and it is now believed that they share common mechanisms of action and pathogenesis, such as impaired autoregulation of the cerebral blood flow and endothelial dysfunction. The presence of clinical and radiologic findings of typical postpartum PLS in our case also complicated our initial diagnosis since we believed that it was a concomitant finding of RCVS.

RCVS is known to occur in patients aged 10-76 years ${ }^{2}$ with a peak in the early forties. A thunderclap headache, the most principal clinical manifestation of RCVS, is characterized by rapid peak intensity within a couple of minutes that lasts from minutes to hours. Such headaches show a recurrent and episodic manner but usually exhibit a self-limiting course within 4 weeks. Less than $10 \%$ of RCVS cases feature mild or no headaches at the time of diagnosis. Seizure episodes are encountered by $1-17 \%$ of affected patients but normally do not advance to persistent epilepsy. Stroke-like symptoms, such as deficits of visual, sensory, or motor systems, occur widely in $10-60 \%$ of RCVS patients and the majority of those events recovered shortly.

MMD has bimodal peaks of onset in childhood and middle age. ${ }^{3}$ The most common clinical presentation is stroke in $30-40 \%$ of adult MMD cases, and hemorrhagic stroke has a higher preponderance in adult than child patients. Seizures are relatively less frequent, showing an incidence of approximately $5 \%$ in adult MMD patients. Therefore, if the initial clinical presentation is nonspecific such as simple headache or seizure in young adult patients with intracranial vascular stenosis, it may be fairly difficult to distinguish between the two disorders. A widely known MRA finding of RCVS is the "sausage-on-a-string sign," which features a smooth, tapering arterial constriction and subsequent dilatation. This is a widespread appearance observed from the proximal circle of Willis (15-50\%) to the secondor third-order branches (>50\%); that is, it occurs even more frequently in distal areas. ${ }^{4}$ In contrast, the affected vessels in MMD are predominantly those that arise from the distal ICA to the proximal MCA and ACA regions, and sequential formation of basal collateral vessels might be noticeable in the differentiation between MMD and RCVS.

Medical conditions being implicated with PLS include hypertension, renal disease, eclampsia, vasculitis, and immunosuppressive agents. Although an elevated postpartum blood pressure as seen in preeclampsia or eclampsia is a well-known clinical finding associated with .the development of PLS, a normal delivery might feature it as well. ${ }^{5}$ Besides, there have been no reported cases of concomitant PLS and MMD to date in the literature.

The pathophysiology of MMD primarily includes fibrocellular thickening of both the intima and the internal elastic lamina in conjunction with attenuation of the intima media. ${ }^{6}$ Meanwhile, loss of cerebral vascular autoregulation with concomitant endothelial dysfunction leading to abnormal vasoconstriction or extravasation of the intravascular fluid are postulated in RCVS and PLS, respectively. ' Since well-preserved endothelial function is crucial for maintaining autoregulation of the cerebral blood flow, ${ }^{8} \mathrm{MMD}$ might be more vulnerable to concomitant PLS or RCVS because of the disrupted structural and functional actions of the endothelium.

The limitation of the current report includes the diagnostic uncertainty of MMD. Since CTA has a relative lack of accuracy at detecting the existence of collateral circulation, further investigations using conventional angiography or high-resolution MRA are warranted.

Here we reported a confusing case of PLS with hid- 
den MMD mimicking RCVS. A thorough history taking and careful interpretation of the angiography results are crucial, and follow-up imaging at the 12th week is required in cases of suspected RCVS.

\section{REFERENCES}

1. Calado S, Vale-Santos J, Lima C, Viana-Baptista M. Postpartum cerebral angiopathy: vasospasm, vasculitis or both? Cerebrovasc Dis. 2004;18:340-341.

2. Ducros A. Reversible cerebral vasoconstriction syndrome. Lancet Neurol. 2012;11:906-917.

3. Kim JS. Moyamoya disease: epidemiology, clinical features, and diagnosis. J Stroke. 2016;18:2-11.

4. Singhal AB, Topcuoglu MA, Fok JW, Kursun O, Nogueira RG, Frosch MP, et al. Reversible cerebral vasoconstriction syndromes and primary angiitis of the central nervous system: clinical, imaging, and angiographic comparison. Ann Neurol. 2016;79:882-894.

5. Shi F, Shen L, Shi Y, Shi L, Yang X, Jin Z, et al. Posterior reversible encephalopathy syndrome after postpartum hemorrhage and uterine artery embolization: a case report. Medicine. 2017;96:e8973.

6. Hosoda Y, Ikeda E, Hirose S. Histopathological studies on spontaneous occlusion of the circle of Willis (cerebrovascular moyamoya disease). Clin Neurol Neurosurg. 1997;99 Suppl 2:S2O3-S2O8.

7. Chen SP, Fuh JL, Wang SJ. Reversible cerebral vasoconstriction syndrome: an under-recognized clinical emergency. Ther Adv Neurol Disord. 2010;3:161-171.

8. Lavi S, Gaitini D, Milloul V, Jacob G. Impaired cerebral $\mathrm{CO}_{2}$ vasoreactivity: association with endothelial dysfunction. Am J Physiol Heart Circ Physiol. 2006;291:H1856-H1861. 